\title{
Microstructure design of a two phase composite using two-point correlation functions
}

\author{
Brent L. Adams \\ b_I_adams@byu.edu \\ H. Garmestani \\ G. Saheli
}

Follow this and additional works at: https://scholarsarchive.byu.edu/facpub

Part of the Mechanical Engineering Commons

Original Publication Citation

Journal of Computer-Aided Materials Design (24) 11: 13-115

\section{BYU ScholarsArchive Citation}

Adams, Brent L.; Garmestani, H.; and Saheli, G., "Microstructure design of a two phase composite using two-point correlation functions" (2004). Faculty Publications. 1013.

https://scholarsarchive.byu.edu/facpub/1013

This Peer-Reviewed Article is brought to you for free and open access by BYU ScholarsArchive. It has been accepted for inclusion in Faculty Publications by an authorized administrator of BYU ScholarsArchive. For more information, please contact ellen_amatangelo@byu.edu. 


\title{
Microstructure Design of a Two Phase Composite using Two-point Correlation Functions
}

\author{
G. Saheli ${ }^{1}$, H. Garmestani ${ }^{*}$, B. L. Adams ${ }^{2}$ \\ ${ }^{1}$ School of Materials Science and Engineering \\ Georgia Institute of Technology \\ Atlanta, GA \\ ${ }^{2}$ Department of Mechanical Engineering \\ Brigham Young University \\ Provo, Utah, 84602
}

\begin{abstract}
Two-point distribution functions are used here as to introduce "Microstructure Sensitive Design" in two-phase composites. Statistical distribution functions are commonly used for the representation of microstructures and also for homogenization of materials properties. The use of two-point statistics allows the composite designer to include the morphology and distribution in addition to the properties of the individual phases and components. Statistical continuum mechanics is used to make a direct link between the microstructure and properties (elastic and plastic) in terms of these two-point statistical functions. An empirical form of the two-point statistical function is used which allows the construction of a composite Hull. Two different composites (isotropic and anisotropic) are considered and the effect of anisotropy for the prediction of the elastic properties is discussed.
\end{abstract}




\section{Nomenclature}

$\mathrm{p}_{\mathrm{ij}}$ : Two-point probability function

$\mathrm{c}_{\mathrm{ij}}, \mathrm{n}_{\mathrm{ij}}$ : Emprical coefficients used in Corson's probability equation

r: Vecotor connected each two points in the microstructure

$\mathrm{c}_{0}, \theta_{0}:$ Reference values

A: Degree of anisotropy defined for the microstructure

$\mathrm{c}_{\mathrm{ijkl}}$ : Elastic constants

$\mathrm{C}_{\mathrm{ijk} \mathrm{l}}$ : Effective elastic constant (Components)

C: Effective elastic constant (Matrix form)

$\sigma_{i j}, \varepsilon_{i j}:$ Stress and strain

$\langle h\rangle$ : Ensemble average of variable $h$

$\widetilde{h}$ : Deviation component of variable $h$

$a_{i j k l}$ : the local inhomogeneity (Components)

$\mathrm{G}_{\mathrm{ij}}$ : Green's function

$\mathrm{K}_{\mathrm{ijk}}, \mathrm{K}_{\mathrm{ijk}}$ : First and second derivative of Green's function 


\section{Keywords}

Aluminum, Anisotropic, Composite, Degree of anisotropy, Design, Elastic modulus, Elastic constant, Isotropic, Lead, Microstructure, Shear modulus, Volume fraction.

\section{Introduction}

Recently a methodology was developed by Adams et. al. [1] that uses a spectral representation as a tool to allow the mechanical design to take advantage of the microstructure as a continuous design variable. This new approach, called microstructure-sensitive design (MSD) uses a set of Fourier basis functions to represent the microstructure (e.g. single orientations) as the material set [1]. The combination of all these elements of microstructure states can be used to construct the property enclosure for any particular structure. The procedure in this methodology can be summarized in the following:

a- Microstructure representation: The microstructure and its details are represented by a set of Orthogonal Basis Functions $\chi_{n}$.

$$
F\left(\chi_{n}, C_{n}\right)=\sum_{n} C_{n} \chi_{n}
$$

Where $C_{n}$ 's are the coefficients, determined for each individual microstructure.

b- Properties and Constraints: The properties and constraints are represented in the same orthogonal space 


$$
P\left(\chi_{n}, p_{n}\right)=\sum_{n} p_{n} \chi_{n}
$$

c- Coupling: The properties and constraints can represent hyper planes in the property enclosure which is defined as a universe of all variation in the inter relation among several properties for the same microstructure.

d- Designer Materials: Intersection of these planes defines the universe of all materials and microstructure (distributions) appropriate for design. This is similar to how Ashby's Diagrams are being used in design [2].

In the present paper, a similar methodology is developed and applied to a two-phase composite material. The difference is the representation of the composite microstructure using empirical equations for the representation of two-point statistical distribution functions rather than spectral analysis. In a polycrystalline material, each grain is considered as a state and an n-dimensional space is then used for the construction of one point distribution function for polycrystalline materials. However a two-phase composite consists of only two phases and the n-dimensional material is reduced to a two-dimensional state assuming that the anisotropic property within each phase is ignored. It is clear that such a construction that uses volume fraction of the second phase can only present a limited description of the composite. In this paper, two-point correlation functions are used as additional parameters for the description of a composite. Twopoint statistics can incorporate not only the distribution and interaction of the two phases but also information on the shape and morphology of each individual phase.

MSD is presently taking advantage of "texture" in the form of Orientation Distribution Function 
for the representation of polycrystalline materials [1]. Orientation Distribution Function (ODF) is a one-point statistical distribution function that only considers volume fractions (or number fractions) of crystallites with the same orientation. Two-point statistical function can be used as a first order correction to the average representation. Two point correlation functions $[3,4,5,6$, 7, 8, and 9] provide information about near neighbor and far field effects and allow the defect sensitive properties to be incorporated in the analysis. The extension to higher order statistics adds a higher order of dimensionality in the Materials Hull. It also presents two major improvements in the analysis for the calculation of effective properties and the evolution of microstructures $[6,8]$. The composite formulation will be markedly enhanced by the use of two point correlations $[3,10$, and 11$]$.

Recent improvements in electron microscopy and image analysis have led to new techniques for analyzing the structure of polycrystalline materials at the scale of the crystalline grains. Orientation Imaging Microscopy (OIM) provides information on the spatial arrangement of lattice orientations in polycrystalline structures and is based on Kikuchi diffractometry [12]. Measurements of local orientation and misorientation of polycrystalline materials are now possible. For the composite, if the orientation of each phase is ignored, the correlation functions can be measured using imaging techniques (optical, SEM...). The use of OIM for the measurement of orientation for a multiphase composite can introduce a large amount of detail and higher order statistical formulations will be needed to incorporate such information for MSD and microstructure analysis.

In this paper we take advantage of anisotropy in the microstructure of a two-phase composite as 
a parameter for design. The methodology presented here is an application of a framework for "Microstructure Sensitive Design" for the case of composites. The earlier work in this effort concentrated on polycrystalline materials. The main issue within this methodology is the microstructure representation and in the earlier work [1] a one-point statistical function (Orientation Distribution Function) was used in the form of Spherical Harmonics. In the present paper a two-point statistical function representation is used for the representation of the microstructure of a two-phase composite. Such a representation will allow us to link the microstructure to elastic properties. Such a representation will allow us to identify and classify composites based on the desired set of properties (inverse methodology). Finally an example of design criteria is provided and the methodology is then used to identify the composite material which satisfied the required constraints.

\section{Representation of Heterogeneity}

The prediction of mechanical property from the details of the microstructure such as phase, crystalline grain distribution and morphology has received special attention in the mechanics and materials community $[3,4]$. The mathematical description of heterogeneity has received some breakthroughs in the last three decades with the works of Kröner and Beran [13, 14, and 15]. More progress has been achieved to calculate the effective properties by making simple assumptions about the microstructure distribution (random, isotropic, and periodic microstructures) or the shape of the second phase (spherical, ellipsoidal...). These studies have 
relied primarily on the one-point probability functions (number or volume fractions of individual states within the microstructure), which ignored shape and geometric characteristics of the microstructure. It was realized that in order to use the measured materials heterogeneity it is necessary to incorporate two and higher order probability functions. Progress was hindered due to lack of experimental techniques to obtain two and three-point correlation functions. These techniques are now available to measure individual crystalline orientation in polycrystalline materials. Extension of this effort to non-random microstructures requires proper definition of $n$th degree statistical correlation functions. For a detailed description of the theoretical discussion and the derivations please refer to work of Garmestani [6,7,8], Beran [16], and Adams [4,17]. A statistical continuum mechanics approach for both elastic and inelastic deformation of composites was introduced earlier [6,7, and 8]. In this paper, the elastic formulation for the isotropic distribution will be extended to include anisotropy.

For two-phase composite structures, the application of two-point statistics requires two different sets of probability functions: The first set can be chosen to describe the probability distribution functions for the interaction of the two phases. This reduces the problem to a composite formulation ignoring the crystalline phase for each component. The two phases can then be taken as isotropic (or anisotropic) phases and the effect of textures can be incorporated in the anisotropy parameters in the constitutive relations. The second set can consist of the probability distribution functions for the individual crystalline phases. This means incorporating the effect of orientation for each phase.

Based on the arguments presented earlier, the first approach will use the composite formulation 
and develop the property space for the two-phase structure. In this analysis Lame's constants are known for two basis isotropic phases.

\subsection{Two-Point distribution Function}

The statistical details of a microstructure can be represented by an n-point probability distribution function. The volume fractions, f1 and $\mathrm{f} 2$ define the one-point probability distribution function that can be used to give an estimate of the effective properties. The details of the shape and morphology of the microstructure including the interaction of the second phase can only be realized by using higher order distribution functions [3, 10]. A two-point distribution function can be defined as a conditional probability function when the statistics of a three-dimensional vector " $\mathbf{r}$ " is investigated once attached to each set of the random points in a particular microstructure. The exponential form of the distribution function as proposed by Corson has been shown to be appropriate for random microstructures [10]. It is represented as,

$$
P_{i j}(r)=\alpha_{i j}+\beta_{i j} \exp \left\lfloor c_{i j} r^{n_{i j}}\right\rfloor
$$

Where $\mathbf{r}$ is a vector in this equation, however in isotropic case, the probability doesn't depend on the direction and $\mathrm{r}$ is assumed to be a scalar.

For a two-phase composite, $\mathrm{i}$ and $\mathrm{j}$ correspond to phases 1 and 2 . This reduces the number of two point functions to four, $\mathrm{P}_{11}(\mathbf{r}), \mathrm{P}_{12}(\mathbf{r}), \mathrm{P}_{21}(\mathbf{r})$, and $\mathrm{P}_{21}(\mathbf{r})$. Normality relations for a two-phase composite and the statistical limitations $\left(\mathrm{p}_{12}=\mathrm{p}_{21}\right)$ require that only $\mathrm{P}_{11}$ be treated as the independent variable and $\alpha$ and $\beta$ are functions of volume fractions. In Eq. (3) the empirical coefficient $\mathrm{c}_{\mathrm{ij}}$ is a scaling parameter representing the correlation distance and can be reformulated 
into an anisotropic form,

$$
c_{i j}(\theta, a)=c_{i j}^{0}\left(A+(1-A) \sin \left(\theta-\theta_{0}\right)\right.
$$

However, $\mathrm{n}_{\mathrm{ij}}$ can be shown to be equal to 1 for a random structure [18]. A three dimensional form of Eq. (3) can also be introduced. The present form is sufficient when the statistical information is uniform in one dimension for the composite. The three dimensional form requires data from a variety of sections through the sample. In Eq.(4), "A" is a material parameter that represents the degree of anisotropy in a microstructure such that $A=1$ corresponds to an isotropic microstructure. Assuming that two perpendicular sections are chosen for the analysis of a composite, we will be able to calculate two anisotropy parameters in these two sections.

\subsection{Definitions and the Procedure}

To estimate the elastic constants the equilibrium equation has to be solved:

$$
\sigma_{i j, j}=0
$$

The relation between elastic stress and strain can be shown by:

$$
\begin{aligned}
& \sigma_{i j}(x)=c_{i j k l}(x) \varepsilon_{k l}(x) \\
& \left\langle\sigma_{i j}\right\rangle=C_{i j k l}\left\langle\varepsilon_{k l}\right\rangle \\
& \varepsilon_{i j}=(1 / 2)\left(\partial u_{i} / \partial x_{j}+\partial u_{j} / \partial x_{i}\right)
\end{aligned}
$$

Symbol $<\mathrm{h}>$ denotes the ensemble average over grains (phases. components...) at state $\mathrm{h}$. So $\left\langle c_{i j k l}\right\rangle$ is the average of the local stiffness defined as follows: 


$$
\left\langle c_{i j k l}\right\rangle=\left\langle c_{i j k l}(x)\right\rangle=\frac{1}{V} \int_{V} c_{i j k l}(x) d V
$$

The same definition is applicable for stress, strain and compliance. The local moduli and compliance as well as the local stress and strain can be defined as a perturbation from the average (mean) values $<. .>$ by defining a new parameter $\left(\begin{array}{l}\sim \\ . .\end{array}\right)$ as in the following equations:

$$
\begin{aligned}
& c_{i j k l}(x)=\left\langle c_{i j k l}\right\rangle+\widetilde{c}_{i j k l}(x) \\
& s_{i j k l}(x)=\left\langle s_{i j k l}\right\rangle+\widetilde{s}_{i j k l}(x) \\
& \sigma_{i j}(x)=\left\langle\sigma_{i j}\right\rangle+\widetilde{\sigma}_{i j}(x) \\
& \varepsilon_{i j}(x)=\left\langle\varepsilon_{i j}\right\rangle+\widetilde{\varepsilon}_{i j}(x)
\end{aligned}
$$

Where $\tilde{c}_{i j k l}(x), \quad \widetilde{s}_{i j k l}(x), \quad \widetilde{\sigma}_{i j k l}(x)$, and $\widetilde{\varepsilon}_{i j k l}(x)$ are, respectively, the deviation of stiffness, compliance, stress and strain at each point from the average value. The following equations should be always satisfied as a result of statistically homogenous media:

$$
\begin{aligned}
& \left\langle\widetilde{c}_{i j k k l}(x)\right\rangle=0,\left\langle\widetilde{s}_{i j k l}(x)\right\rangle=0 \\
& \left\langle\widetilde{\sigma}_{i j k l}(x)\right\rangle=0,\left\langle\widetilde{\varepsilon}_{i j k l}(x)\right\rangle=0
\end{aligned}
$$

\subsection{Effective Elastic Constants}

Statistical continuum mechanics analysis is used to predict the elastic properties of a composite. The theoretical framework has been developed for isotropic distributions in composites by Garmestani, et. al. [6,7] and for a textured polycrystalline material by Adams et.al. [16, 19]. Here, a brief discussion is provided for the calculation of the effective elastic constants for 
isotropic distribution and will be extended to anisotropic distributions,

Effective Elastic constants "C" of a composite are defined by the equation:

$$
\bar{\sigma}=C \bar{\varepsilon}
$$

Where $\bar{\sigma}$ and $\bar{\varepsilon}$ are the average stress and strain respectively, and $\mathrm{C}$ is the effective elastic constant of the composite. Applying Hill's criteria the effective elastic constants can be written as (for details see paper by Garmestani, et. al. [6, 7]):

$$
C=\langle c\rangle+\langle c a\rangle
$$

Where the first term is the average elastic tensor and the second term is the average deviation of the elastic constants from the mean. The fourth rank tensor $\mathrm{a}=\left(a_{i j k l}\right)$ is introduced here to represent the local inhomogeneity. Therefore the effective property can now be defined by:

$$
C_{i j k l}=\left\langle c_{i j k l}\right\rangle+\left\langle\widetilde{c}_{i j m n}(x) a_{m n k l}(x)\right\rangle
$$

By substituting local stress (Eq. (6)) into the equilibrium Equations (Eq. (5)), an equation for displacement is obtained. Differentiating the equation of displacement and multiplying the result by $c_{i j k l}$, the second term in Eq. (12) will be calculated by:

$$
\left\langle\widetilde{c}_{i j k u}(x) a_{k u r s}(x)\right\rangle=\int_{V} \partial\left[K_{k p u}\left(x, x^{\prime}\right)\left\langle\widetilde{c}_{i j k u}(x) \widetilde{c}_{p m r s}\left(x^{\prime}\right)\right\rangle\right] \partial \partial x_{m}^{\prime} d X^{\prime}-\int_{V} K_{k p u m}\left(x, x^{\prime}\right)\left\langle\widetilde{c}_{i j k u}(x) \widetilde{c}_{p m r s}\left(x^{\prime}\right)\right\rangle d X^{\prime}
$$

Where $\mathrm{x}$ and $\mathrm{x}^{\prime}$ are two different position in the media, and $\mathrm{dX}^{\prime}$ shows the volume integral on the volume element around the position $\mathrm{x}^{\prime}$.

In which the correlation function is defined by: 
$\left\langle\widetilde{c}_{i j k u}(x) \widetilde{c}_{p m r s}\left(x^{\prime}\right)\right\rangle=\widetilde{c}_{1 j k k} \widetilde{c}_{1 p m r s} p_{11}+\widetilde{c}_{1 j k k} \widetilde{c}_{2 p m r s} p_{21}+\widetilde{c}_{1 j k k} \widetilde{c}_{2 p m r s} p_{12}+\widetilde{c}_{2 i j k u} \widetilde{c}_{2 p m r s} p_{22}$

And:

$$
\begin{aligned}
& K_{k p u}=\left(G_{k p, u}+G_{u p, k}\right) / 2 \\
& K_{k p u m}=\left(G_{k p, u m}+G_{u p, k m}\right) / 2
\end{aligned}
$$

Where $\mathrm{G}$ is the Green's function used for solving the equilibrium equations. For definition of Green's function for isotropic and anisotropic cases, readers should refer to [7, 19].

\section{Results and Discussions: Composite Property Enclosure}

In this section the elastic properties are calculated for two types of composites. First an isotropic composite with a randomly distributed second phase is considered. In such a composite, the probability distribution functions are isotropic and independent of orientation. In this case the probability functions in Eq. (3) are sufficient to characterize the microstructure. Next a special case of an anisotropic composite is considered such that the microstructure of any section perpendicular to a particular direction has the same statistics. The anisotropy is then considered in only two sections of the composite. In the simulation of this microstructure, the probability distribution function changes with orientation and magnitude of the vector " $\mathbf{r}$ " on each section. The measurements of this composite on any section perpendicular to one particular direction provides the same statistical information within which the statistics maybe anisotropic. 


\subsection{Isotropic Distribution}

For a randomly distributed isotropic composite, the correlation functions are independent of orientation and can be taken out of the integral in the second term of Eq. (13). The integrand then only includes the Green's function and has to be integrated over the boundary of a sphere. It is proved that this integral goes to zero which means there is no contribution from the twopoint statistics for an isotropic material and only the first integral or the one-point statistics (volume fractions) contributes to effective elastic property. The two substituting phases are Aluminum and Lead with Lame's constants of $(\lambda=64.286, \mu=25)$ and $(\lambda=25.88, \mu=4.926)$, respectively. The effective elastic modulus for an isotropic distribution is plotted as a function of volume fraction in Fig (1) and Fig (2). Several models have been studied to approximate the properties of heterogeneous materials $[20,21,22,23]$ here Voigt upper bound and Ruess lower bound are calculated and shown in the graphs for a comparison with simulation results. Voigt assumes a uniform strain, and Reuss assumes uniform stress in both phases. [24, 25]. In general, upper bound and lower bound for some components of stiffness and compliance can be shown by the following equations:

$$
\begin{aligned}
& C_{i i j i}^{u p p e r}=f_{1} C_{i i i i}^{1}+f_{2} C_{i i i i}^{2} \\
& C_{i j i j}^{u p p e r}=f_{1} C_{i j i j}^{1}+f_{2} C_{i j i j}^{2} \\
& C_{i i i i}^{\text {lower }}=\frac{C_{i i i i}^{1} C_{i i i i}^{2}}{f_{1} C_{i i i i}^{2}+f_{2} C_{i i i i}^{1}} \\
& C_{i j i j}^{\text {lower }}=\frac{C_{i j i j}^{1} C_{i j i j}^{2}}{f_{1} C_{i j i j}^{2}+f_{2} C_{i j i j}^{1}}
\end{aligned}
$$


Where $C_{i i i i}^{1}, C_{i i i i}^{2}, C_{i j i j}^{1}$, and $C_{i j i j}^{2}$ are diagonal terms of the forth rank elastic stiffness tensor for phase 1 and 2, and $C_{i i i i}^{u p p e r, l o w e r}$ and $C_{i j i j}^{u p p e r, l o w e r}$ are upper and lower bounds.

$\{$ Insert Fig(1) and (2)\}

Fig (1) shows the variation of the elastic modulus for $\mathrm{Al}-\mathrm{Pb}$ composite for different volume fractions of Aluminum. It illustrates that the statistical model provides a good estimate for the elastic properties. The predictions of the statistical model seem to be closer to the upper bound for larger volume fractions and closer to the lower bound for smaller volume fractions. The difference between the predictions and the upper bound decreases from $80 \%$ to $13 \%$ as the second phase volume fraction increases. The statistical predictions for the shear modulus of the composite (commonly known as $\mu$ ) are shown in Fig (2). Although the composite is considered to be isotropic, three elastic coefficients $\left(\mathrm{C}_{1111}, \mathrm{C}_{1122}\right.$, and $\left.\mathrm{C}_{1212}\right)$ can be independently predicted for this simulation. The shear modulus, $(\mu)$ can be calculated directly from the simulation as $\mathrm{C}_{1212}$. It can also be predicted from $\mathrm{C}_{1111}$ and $\mathrm{C}_{1122}$ through the isotropic relation for $\mu$.

$$
\mu=(1 / 2)\left(C_{1111}-C_{1122}\right)
$$

These two values should be identical for an isotropic composite $\left(\mu=C_{1212}\right)$; whereas, in this simulation the probability distributions for the two phases will determine whether this is valid. The simulations show that the values obtained from $C_{1212}$ are very close to the upper bound. The differences between these calculated values and the upper bound is less than $4 \%$. However the values obtained from $(1 / 2)\left(C_{1111}-C_{1122}\right)$ are better estimates for the shear modulus $(\mu)$ of the 
composite. It is observed that the results of the simulation are very close to the upper bound for the larger volume fractions of aluminum, and closer to the lower bound for smaller values of volume fractions. The statistical values of $\mathrm{E}$ and $\mu$ are also compared to Self-Consistent model [26] and they show good agreement with the model. The largest difference for both $\mathrm{E}$ and $\mu$ is about $10 \%$.

\subsection{Anisotropic Distribution}

The effect of anisotropy is examined here by considering a special type of a two-phase composite that gives the same anisotropic distribution in every plane perpendicular to a particular direction (Z-direction). This means that the three-dimensional distribution function can be measured to be identical from any plane normal to this direction. The two individual phases of the composite are considered to be isotropic.

As it was mentioned before volume fraction and the degree of Anisotropy (A) are considered as two design parameters in this work. In this section the degree of Anisotropy is calculated for 3 samples of $\mathrm{Al}-\mathrm{Pb}$ composite by having the distribution of $\mathrm{p}_{11}$. The volume fraction of $\mathrm{Al}$ in the samples is $20 \%, 30 \%$, and $40 \%$ respectively. As an example the fitted curve through modified Corson's equation for the case of $30 \%$ is shown in Fig. (3). The values of $C_{1111}, C_{2222}, C_{3333}$, and $C_{1133}$ are calculated for the three samples and the effect of degree of anisotropy on property is studied in transverse plane. In Fig(4) the variation of anisotropy is shown for different values of A for the case of vol2 $=30 \%$. It's observed that as $\mathrm{A}$ gets closer to $1, \mathrm{C}_{1111}$ gets closer to $\mathrm{C}_{2222}$ 
which corresponds to an isotropic distribution in transverse plane.

$\{$ Insert Fig(3),(4), and (5)\}

Fig. (5) illustrates the property enclosure of the composite $\mathrm{Al}-\mathrm{Pb}$. Each point in this enclosure represents a microstructure distribution with a specific volume fraction and specific anisotropy "A". Two elastic coefficients of the composite are shown for three different volume fractions $(20 \%, 30 \%$ and $40 \%)$ of $\mathrm{Al}$.

In Table (1) the effective elastic coefficients $C_{1111}$ and $C_{3333}$ of the composite are also calculated for three samples. In this particular microstructure, Z-direction may be considered such that the elastic properties in that direction, $C_{3333}$, is smaller than $C_{1111}$. It's also evident that although the two phases are isotropic, the statistical model results in an anisotropic behavior for the elastic modulus.

The contribution of the different higher order statistical terms for the calculation of $C_{1111}$ and $C_{3333}$ is also shown in this Table. Term 2 is the contribution from the two-point statistical functions that are included in the second integral equation of Eq. (13). This contribution is 15\% to $27 \%$ in calculation of $C_{1111}$. For the case of $C_{3333}$, the contribution of the second term is between $31 \%$ and $47 \%$. As it was noted before, the second term does not contribute for the case of isotropy and is only observed in the anisotropic case. 


\subsection{Composite Design}

To illustrate the use of the present methodology in composite design, an example is given for a certain design project requiring a knowledge of the variations in the ratio of the elastic moduli $\mathrm{C}_{3333} / \mathrm{C}_{1111}$. The composite system will be limited to the one discussed in the previous sections $(\mathrm{Al}-\mathrm{Pb})$. Let's consider a certain design in which the ratio of the elastic moduli $\mathrm{C}_{3333} / \mathrm{C}_{1111}$ needs to be minimized. The composite in this design project is quantified using the two-point statistical functions defined in equations 3 and 4 . The design variables are now defined based on two parameters: volume fraction and degree of anisotropy as the representation of one and two point functions. Let us consider the example above and for the purpose of illustration, the three microstructures above are considered. It is clear that these three microstructures can be extended to a large set of microstructures by varying A and the volume fraction of the second phase. The connection can be set up as an analytical tool for design using the homogenization relations explained above. Calculating the ratio of $\mathrm{C}_{3333} / \mathrm{C}_{1111}$ for different values of vol (Al) and $\mathrm{A}$ (degree of Anisotropy), the statistical analysis above shows that for any given values of $\mathrm{A}$, the composite has the lowest ratio of the longitudinal elastic property with respect to transverse elastic property at $\operatorname{vol}(\mathrm{Al})=30 \%$ (Fig.6). It means this methodology can be used to predict the microstructure in a specific design. The design constraints would lead us to a set of optimized properties as needed. The microstructure of the composite is predicted in terms of the statistical parameters (here as volume fractions and degree of anisotropy factor). However this microstructure is not unique. For instance for this case, having vol( $\mathrm{Al})=30 \%$ and $\mathrm{A}$ (degree of anisotropy $)=0.0258$, there are a variety of microstructures that ensure this specification. 
Meanwhile, knowing these two parameters limits the microstructure to a subset of microstructures with a specific volume fraction and degree of anisotropy. Therefore two parameters defined in this section are adequate to represent the microstructure needed for design.

\section{Conclusions}

Microstructure Sensitive Design has been applied to a two-phase composite. The key to MSD is the correct representation of the microstructure. Here a simplified empirical form of the twopoint probability function is used for the microstructure representation instead of the spectral representation. The statistical formulation uses the two-point statistical functions to incorporate the effect of the microstructure distribution. The results show that in the case of isotropic distributions the two-point statistics will not contribute to the effective properties but the statistical analysis can provide a better estimate for the effective properties. In the case of anisotropic distribution, the two-point function can introduce anisotropy in the effective elastic properties. Such anisotropy can be used as a parameter to engineer new composites with an imposed distribution. These parameters and their concomitant properties are considered to be continuous design variables that can be used for optimization of composites. An example is provided such that the design constraint can be reduced to a set of microstructures. The design objectives and constraints are then communicated by specific iso-property surfaces in this space. 


\section{Acknowledgement}

This work has been funded under the AFOSR grant \# F49620-03-1-0011 and Army Research Lab contract \# DAAD17-02-P-0398 and DAAD 19-01-1-0742.

\section{Reference}

[1] Adams, B.L., Lyon, M.B., Kalidindi, S.R., and Garmestani, H., Materials Science Forum, 408-412 (2002) 493.

[2] Ashby, M.F., Metall Trans A., 14 (1983) 1755.

[3] Torquato, S., Stell, G., J. Chem. Phys. 77 (1982) 137.

[4] Adams, B.L., Morris, P.R., Wang, T.T., Willden, K.S., and Wright, S.I., Acta Metall., 35 (1987) 2935.

[5] Torquato, S., Physical review Letters.,79 (1997) 681.

[6] Garmestani, H., Lin, S., and Adams, B.L., Int. J. Plasticity, 14 (1998) 719.

[7] Garmestani, H., Lin, S., Journal of Composites: Part B., 31 (2000) 39.

[8] Garmestani, H., Lin, S., Adams, B., and Ahzi, S.I, Journal of the Mechanics and physics of Solids, 49 (2001) 589.

[9] Lin, S., Garmestani, H., and Adams, B., International journal of solids and structures, 37 (2000) 423. 
[10] Corson, P. B., J. Applied Physics, 45 (1974) 3159.

[11] Corson, P.B., J. Applied Physics, 45 (1974) 3165.

[12] Garmestani, H., Kalu, P., and Dingley, D., Journal of Materials Science and Engineering A. 242 (1998.) 284.

[13] Kroner, E., Statistical Continuum Mecahnics, Springer verlag, Wien., NY, 1972.

[14] Kröner, E., J. Mech. Phys. Solids, 25 (1977) 137.

[15] Beran, M. J., Statistical Continuum Theories. Interscience Publishers, NewYork, 1968

[16] Beran, M.J., Mason, T.A., Adams, B.L., and Olsen, T.,., J. Mech. Phys. Solids, 44 (1996) $1543-1563$.

[17] Adams, B.L., Canova, G.R., and Molinari, A., Textures and Microstructures, 11 (1989) 57.

[18] Tewari, A., Gokhale, A.M., Spowart, J.E., Miracle, D.B., Acta Materiala 52 (2003) 307.

[19] Mason, A.T., Adams, B..L., Metallurgical and Materials transactions A 30 (1999) 969.

[20] Ostaja-Starzewski, M., Int. J. Structures., 35 (1998) 2429.

[21] Torquato, S., Ann. Rev. Matr. Res. 32 (2002)

[22] Pande, C. S., Acta Metallurgica, 36 (1988) 1261.

[23] Pande, C. S., Dantsker, E., Acta Metall., 42 (1994) 2899.

[24] Voigt, W., Wied. Ann., 38 (1889) 573.

[25] Reuss, A., Math. Mech., 9 (1929) 49. 
[26] S. Nemat-Nasser, Micromechanics: Overall properties of heterogeneous materials, Elsevier, 1999.Netherlands.

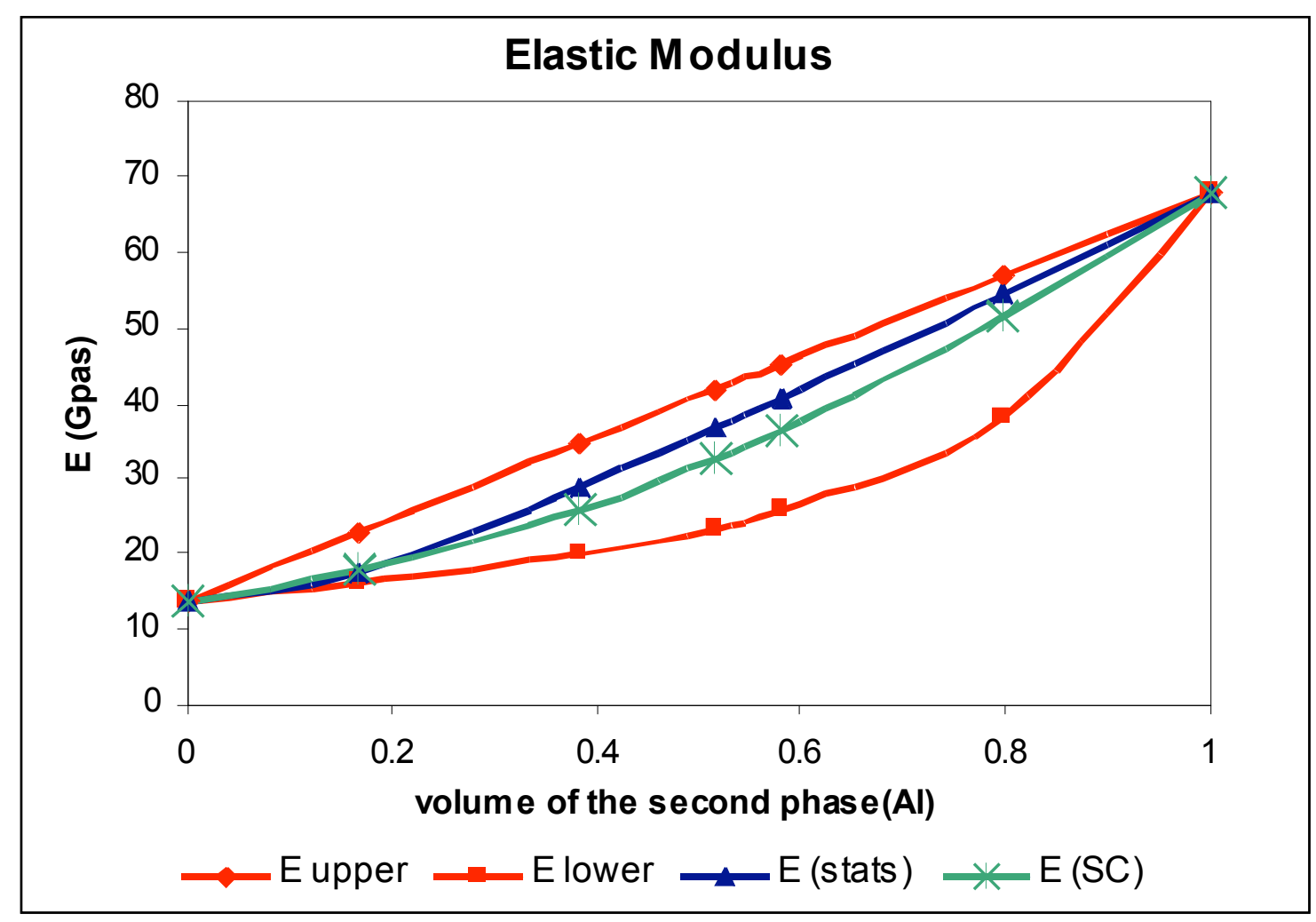


Fig (1). Effective elastic modulus of Al-Pb v.s. volume fraction of the second phase 


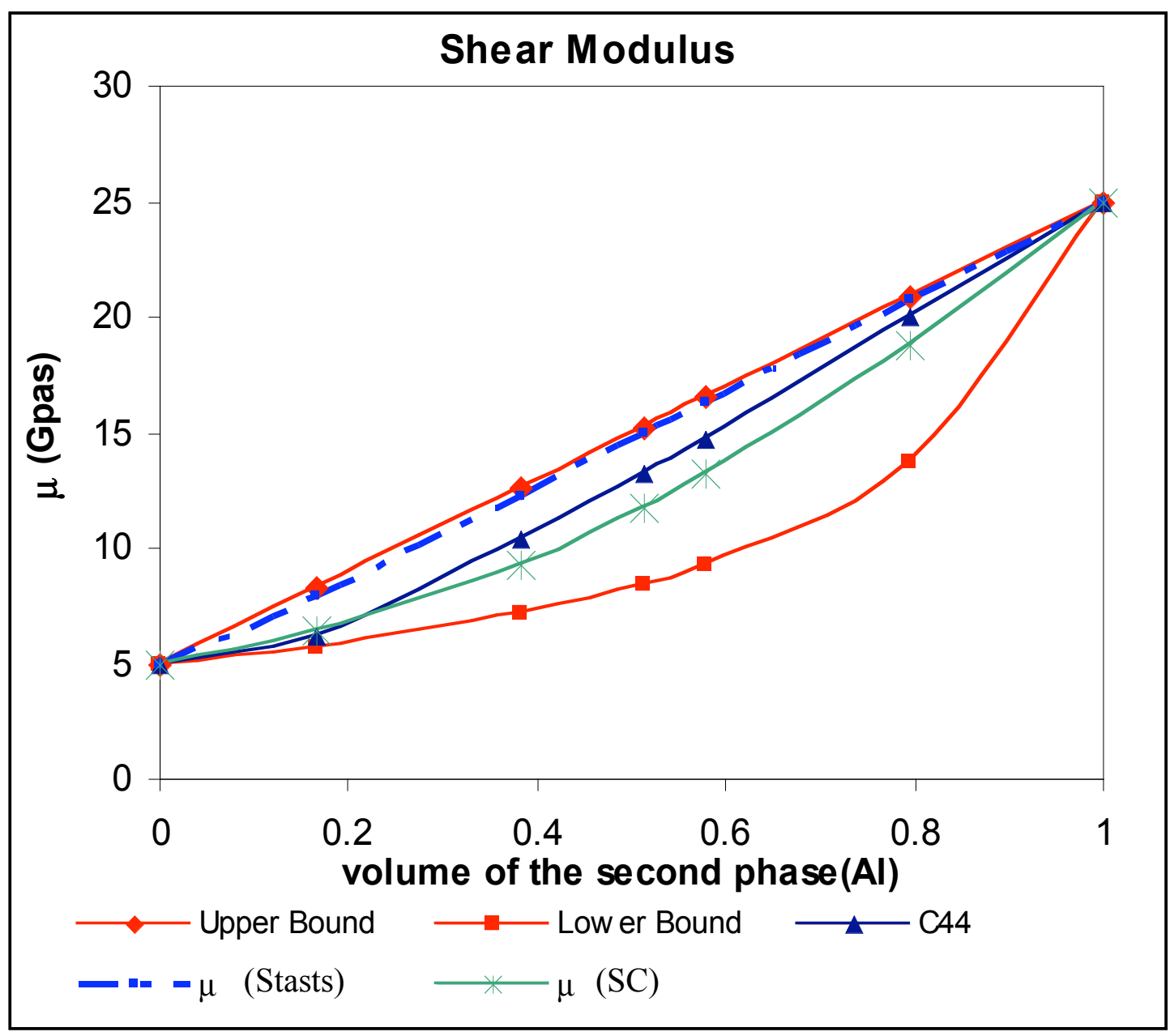

Fig (2). Effective elastic shear modulus of Al-Pb v.s. volume fraction of the second phase: In this graph, the values of $\mu$ is calculated from the isotropic relation for $\mu$ (Eq.(17)). $\mathrm{C}_{1122}$ and $\mathrm{C}_{1111}$ in this relation are the statistical values. Also, the values of $\mathrm{C}_{1212}$ shown in the graph are directly calculated from the simulation. 


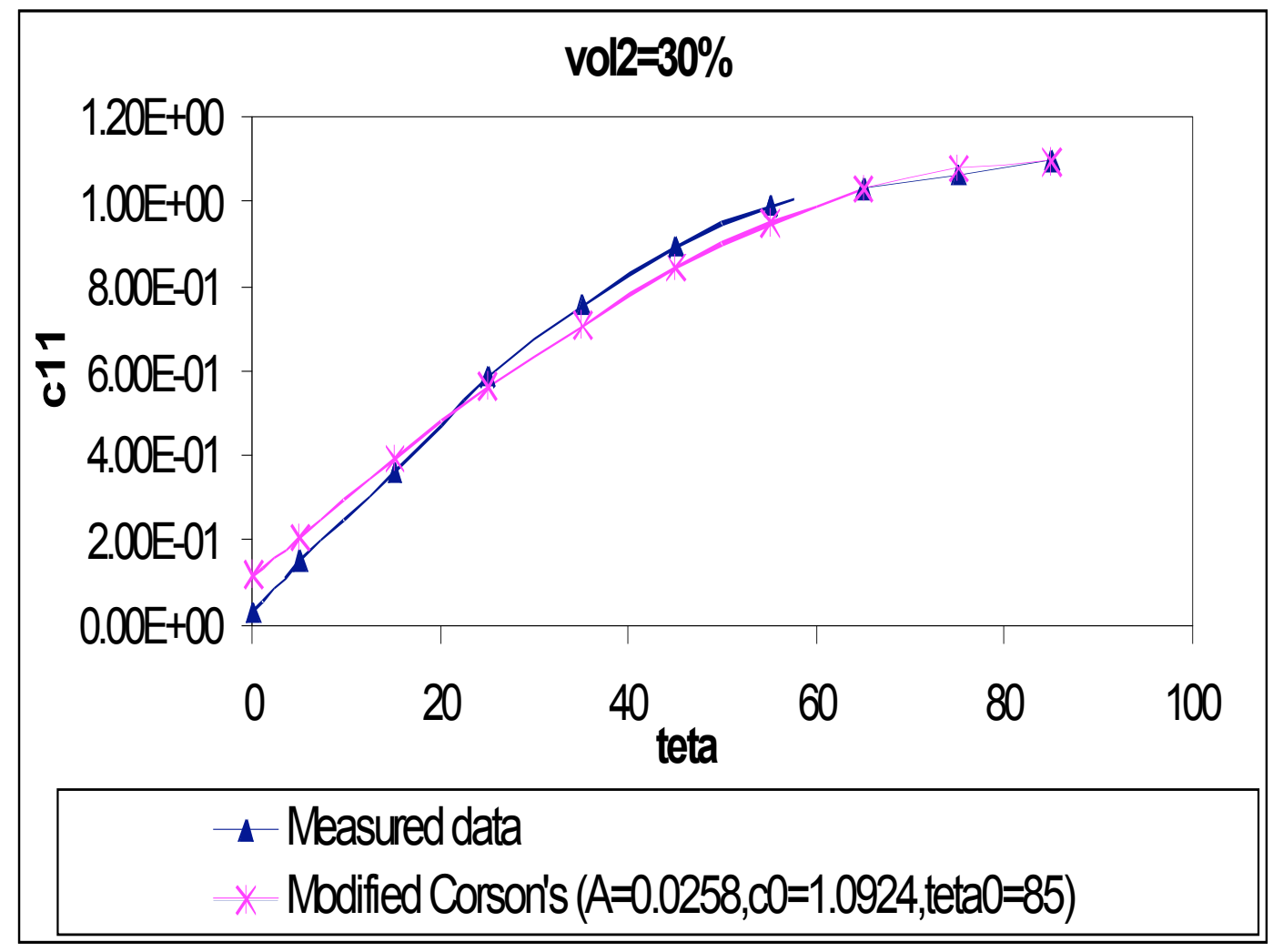

Fig.(3). Modified Corson's equation fitted to measured values of $\mathrm{p}_{11}$ 


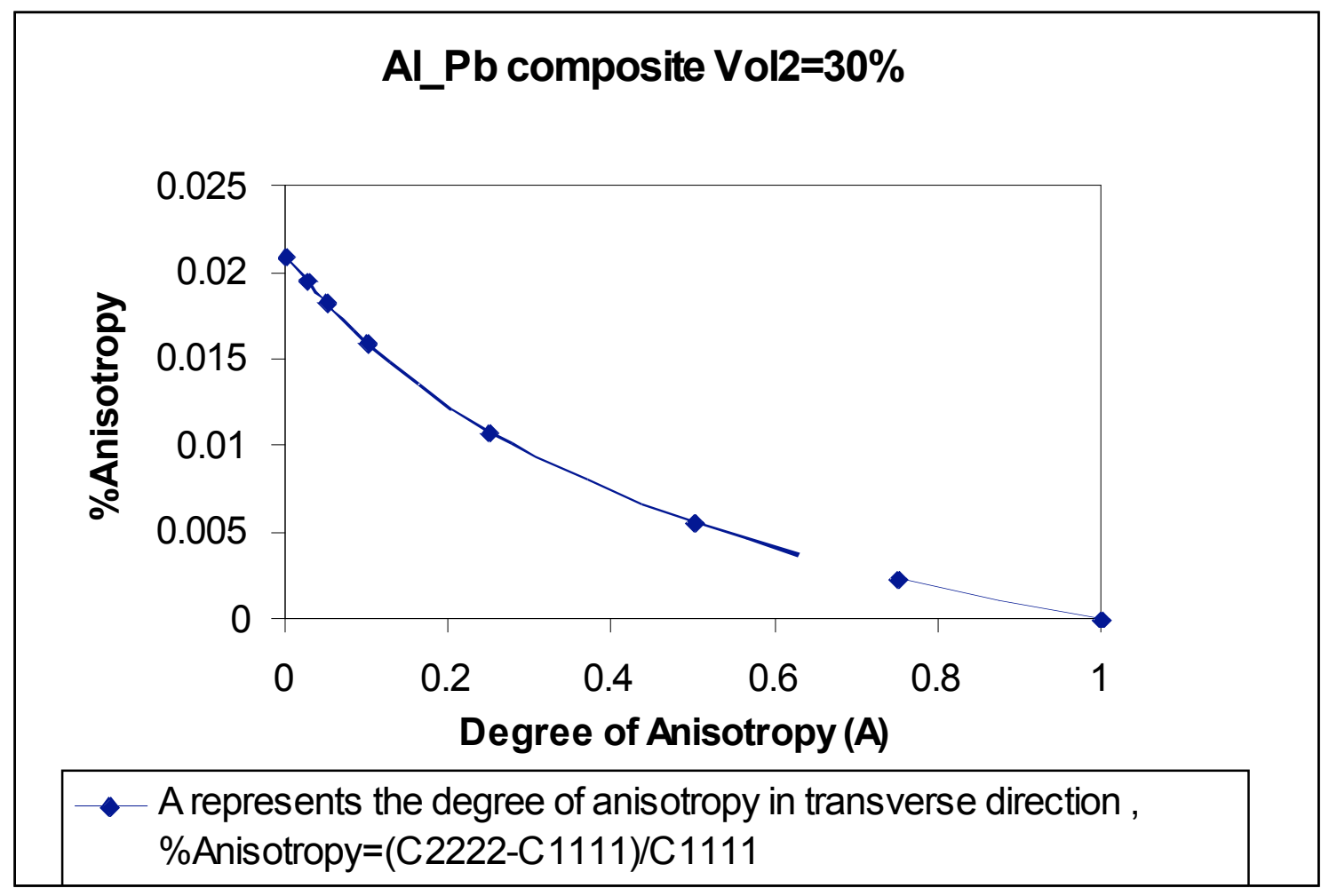

Fig.(4) Variation of anisotropy for different values of A 


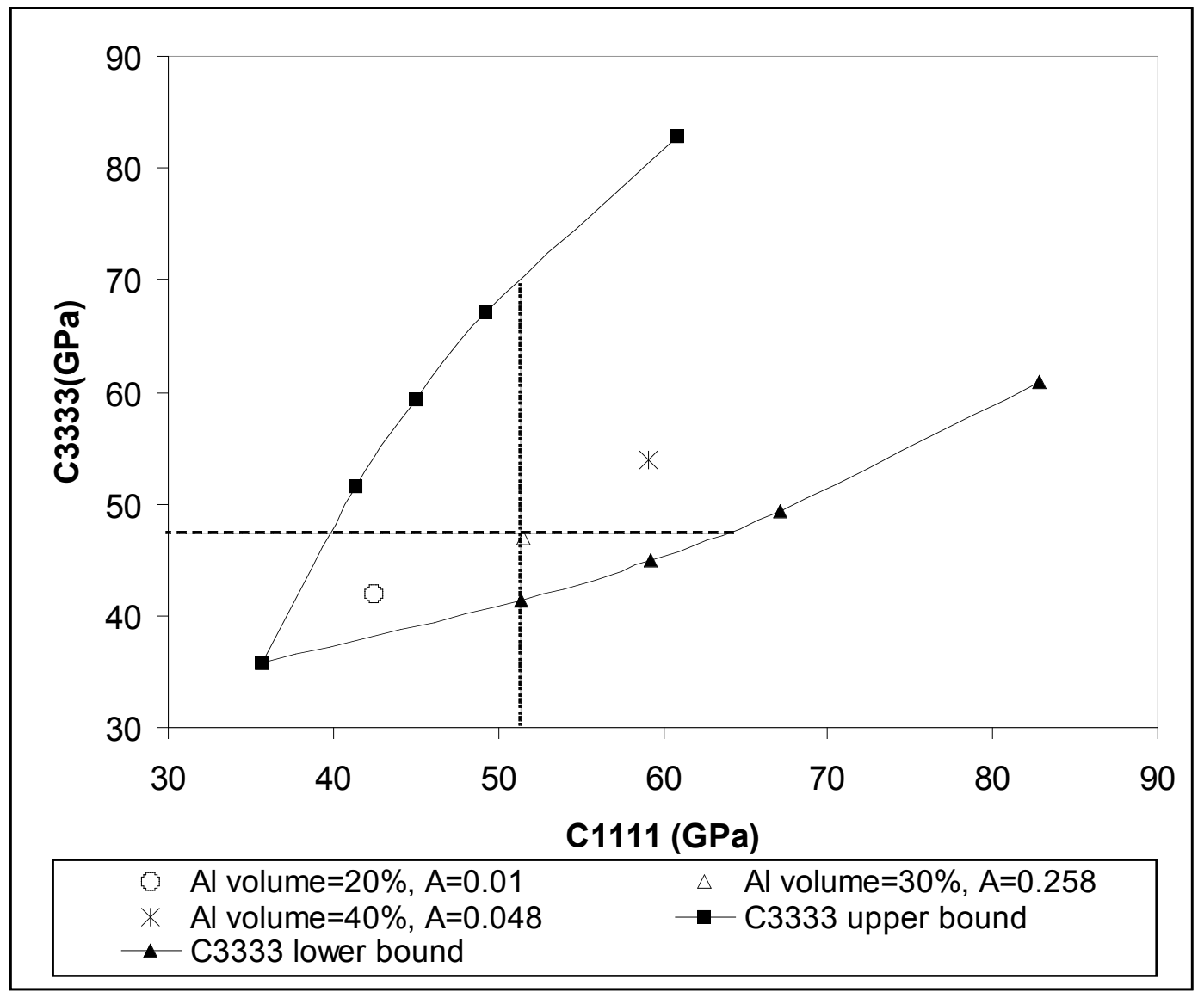

Fig (5). Property enclosure of anisotropic composite Al-Pb 


\begin{tabular}{|c|c|c|c|c|c|c|c|r}
\hline$v$ of Al & Upper Bound & $C_{1111}$ & Term1 & Term2 & $C_{3333}$ & Term1 & Term2 & Lower Boun \\
\hline $20 \%$ & 51.45 & 42.47 & -6.58 & 2.39 & 41.89 & -6.58 & 2.97 & 41.43 \\
\hline $30 \%$ & 59.30 & 51.59 & -6.49 & 1.20 & 46.99 & -6.49 & 5.82 & 45.02 \\
\hline $40 \%$ & 67.15 & 59.129 & -5.85 & 2.17 & 53.92 & -5.85 & 7.37 & 49.28 \\
\hline
\end{tabular}

Table (1). The contribution of one-point and two-point correlation statistics in the calculation of effective elastic modulus $\mathrm{C}_{1111}$ and $\mathrm{C}_{3333}$ for anisotropic $\mathrm{Al}-\mathrm{Pb}$ 


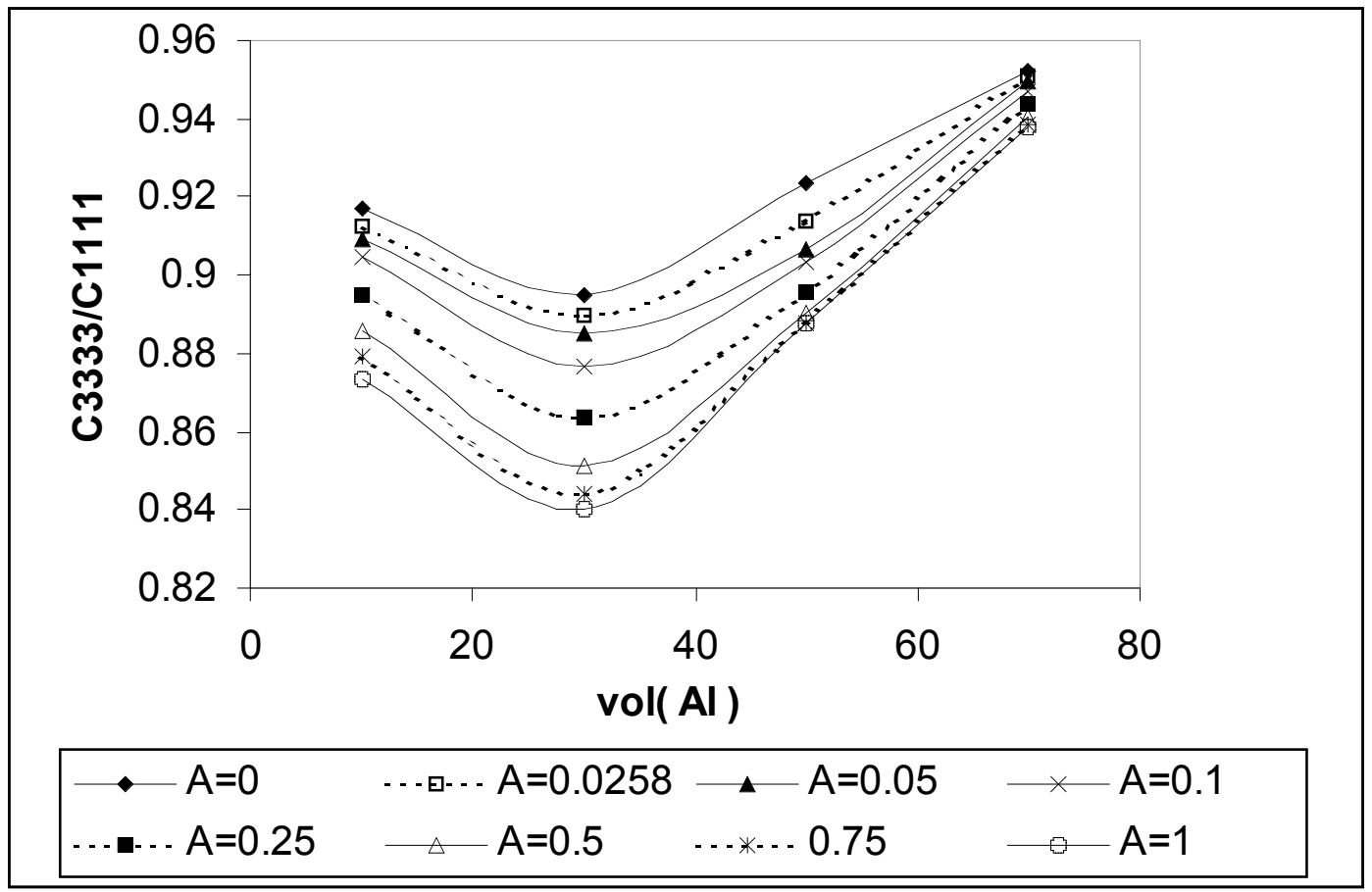

Fig (6). Composite Design: Minimizing the longitudinal /transverse property of anisotropic composite Al$\mathrm{Pb}$ ( A represents the degree of Anisotropy in transverse direction) 